\title{
Rheumatoid arthritis in the Middle East and Africa: are we any closer to optimising its management?
}

\author{
Jamal Al Saleh • Gaafar Ragab • Peter Nash • Hussein Halabi • \\ Ahmed Laatar • Ali M. El-Sayed Yousef • Hamdi Ehsouna • \\ Mohammed Hammoudeh
}

Received: 15 October 2014 / Revised: 24 October 2014 / Accepted: 26 October 2014 / Published online: 7 November 2014

(C) International League of Associations for Rheumatology (ILAR) 2014

\section{Introduction}

A recent editorial considered the management of rheumatoid arthritis (RA) in the Middle East and Africa [1]. Following review of the limited available evidence in the literature specifically that is from this region, it was suggested that management of RA is suboptimal for a variety of reasons [1]. The editorial authors met to determine whether the European League Against Rheumatism (EULAR) consensus recommendations published in 2010 [2] were applicable and appropriate for implementation in

\author{
J. Al Saleh $(\bowtie)$ \\ Rheumatology Section, Dubai Hospital, Dubai, United Arab \\ Emirates \\ e-mail: drjamalalsaleh@hotmail.com \\ G. Ragab \\ Cairo University, Cairo, Egypt \\ P. Nash \\ Department of Medicine, University of Queensland, Brisbane, QLD, \\ Australia \\ H. Halabi \\ King Faisal Specialist Hospital \& Research Centre, Riyadh, Saudi \\ Arabia \\ A. Laatar \\ Department of Rheumatology, University of Tunis El Manar, Tunis, \\ Tunisia \\ A. M. El-Sayed Yousef \\ Department of Rheumatology and Immunology, Benha Teaching \\ Hospital, Benha, Egypt \\ H. Ehsouna \\ Faculty of Medicine, Benghazi University, Benghazi, Libya \\ M. Hammoudeh \\ Department of Medicine, Hamad General Hospital, Doha, Qatar
}

the MENA region and South Africa [1]. The group made recommendations on next steps to improve the management of RA in this region, including collection of epidemiological data to elucidate better the prevalence, severity and burden of RA in this region; educational initiatives to raise awareness of the disease and dispel misconceptions among health care professionals (HCPs) and patients; development of regional guidelines to increase implementation of an evidence-based approach and improve outcomes; and facilitation of access to treatments in line with the recommendations [1]. In addition, locally relevant issues not commonly seen in Europe such as high rates of hepatitis $\mathrm{B}$ and $\mathrm{C}$, tuberculosis (TB) and parasitic infections as well as access and monitoring difficulties should be considered.

\section{New EULAR guidelines: raising the bar further}

Towards the end of 2013, revised EULAR guidelines were published [3]. The overarching principles behind the updated recommendations remain broadly the same, although the order of priority has been changed and the final principle has some minor material alterations. Table 1 highlights the differences between the previous and current EULAR recommendations, while Fig. 1 highlights the differences between the previous and current EULAR treatment algorithms.

\section{Is management of RA in the Middle East and Africa any closer to the EULAR ideal?}

Following the publication of the 2013 EULAR recommendations, it is opportune to assess whether any advances have been made in the management of RA in the Middle East and Africa. We therefore undertook a similar survey to that 
Table 1 European League Against Rheumatism (EULAR) treatment recommendations [2, 3]

\begin{tabular}{l} 
2010[2] \\
\hline A. Rheumatologists are the specialists who should primarily care for \\
patients with RA \\
B. Treatment of patients with RA should be based on a shared decision \\
between the patient and the rheumatologist \\
C. RA is expensive in regards to medical costs and productivity costs, \\
both of which should be considered by the treating rheumatologist
\end{tabular}

hing principles

2013[3]

A. Treatment of RA patients should be based on a shared decision

between the patient and the rheumatologist

B. Rheumatologists should primarily care for RA patients

C. RA incurs high individual, societal and medical costs, all of which should be considered in its management by the treating rheumatologist

\section{Recommendations}

1. Treatment with synthetic DMARDs should be started as soon as the diagnosis of RA is made

1. Therapy with DMARDs should be started as soon as the diagnosis of RA is made

2. Treatment should be aimed at reaching a target of remission or low disease activity as soon as possible in every patient;

2. Treatment should be aimed at reaching a target of remission or low disease activity in every patient

as long as the target has not been reached, treatment should be adjusted by frequent (every 1-3 months)-and strict

3. MTX should be part of the first treatment strategy in patients with active RA

4. When MTX contraindications (or intolerance) are present, the following DMARDs should be considered as part of the (first) treatment strategy: leflunomide, sulfasalazine or injectable gold

5. In DMARD-naïve patients, irrespective of the addition of glucocorticoids, synthetic DMARD monotherapy father than combination therapy of synthetic DMARDs may

6. Glucocorticoids added at low hich doses to synthetic DMARD monotherapy (or combinations of synthetic DMARDs) provide benefit as initial short term treatment but should be tapered as rapidly as clinically feasible

7. If the treatment target is not achieved with the first DMARD strategy, addition of a biologic DMARD should be considered when poor prognostic factors are present; in the absence of poor prognostic factors, switching to another synthetic DMARD strategy should be considered

8. In patients responding insufficiently to MTX and/or other synthetic DMARDs with or without glucocorticoids, biologic DMARDs should be started; current practice would be to start a TNF inhibitor (adalimumab, certolizumab, etanercept, golimumab, infliximab) which should be combined with MTX

9. Patients with RA for whom a first TNF inhibitor has failed should

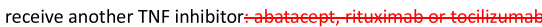

10. In cases of refractory severe RA or contraindications to biologic agents or the previously mentioned synthetic DMARDs, the following synthetic DMARDs might be also considered, as monotherapy or in combination with some of the above: azathioprine or ciclosporin A (or, exceptionally, cyclophosphamide)

11. Intensive medication strategies should be considered in every patient, although patients with poor prognostic factors have more to gain

12. If a patient is in persistent remission, after having tapered glucocorticoids, one can consider tapering biologic DMARDs, especially if this treatment is combined with a synthetic DMARD

13. In cases of sustained long-term remission, cautious titration of synthetic DMARD dose could be considered, as a shared decision between patient and doctor

14. DMARD-naive patients with poor prognostic markers might be considered for combination therapy of methotrexate plus a biologic agent

15. When adjusting treatment, factors apart from disease activity, such as progression of structural damage, comorbidities and safety concerns, should be taken into account

3. Monitoring should be frequent in active disease (every 1-3 months); if there is no improvement by at most 3 months after the start of treatment or the target has not been reached by 6 months, therapy should be adjusted

4. MTX should be part of the first treatment strategy in patients with active RA

5. In cases of MTX contraindications (or intolerance), sulfasalazine or leflunomide should be considered as part of the treatment strategy

6. In DMARD-naïve patients, irrespective of the addition of glucocorticoids, conventional synthetic DMARD monotherapy or combination therapy of csDMARDs should be used

7. Low-dose glucocorticoids should be considered as part of the initial treatment strategy (in combination with one or more conventional synthetic DMARDs) for up to 6 months, but should be tapered as rapidly as clinically feasible

8. If the treatment target is not achieved with the first DMARD strategy, in the absence of poor prognostic factors, change to another conventional synthetic DMARD strategy should be considered; when poor prognostic factors are present, addition of a biologic DMARD should be considered

9. In patients responding insufficiently to MTX and/or other conventional synthetic DMARD strategies, with or without glucocorticoids, biologic DMARDs (TNF inhibitors [adalimumab, certolizumab pegol, etanercept, golimumab, infliximab, biosimilars (as approved according to a thorough approval process, such as by EMA and/or FDA)], abatacept or tocilizumab, and, under certain circumstances, rituximabt) should be commenced with MTX

10. If a first biologic DMARD has failed, patients should be treated with another biologic DMARD; if a first TNF inhibitor therapy has failed patients may receive another TNF inhibitor [adalimumab, certolizumab pegol, etanercept, golimumab, infliximab, biosimilars (as approved according to a thorough approval process, such as by EMA and/or FDA)] or a biological agent with another mode of action

11. Tofacitinib may be considered after biological treatment has failed 

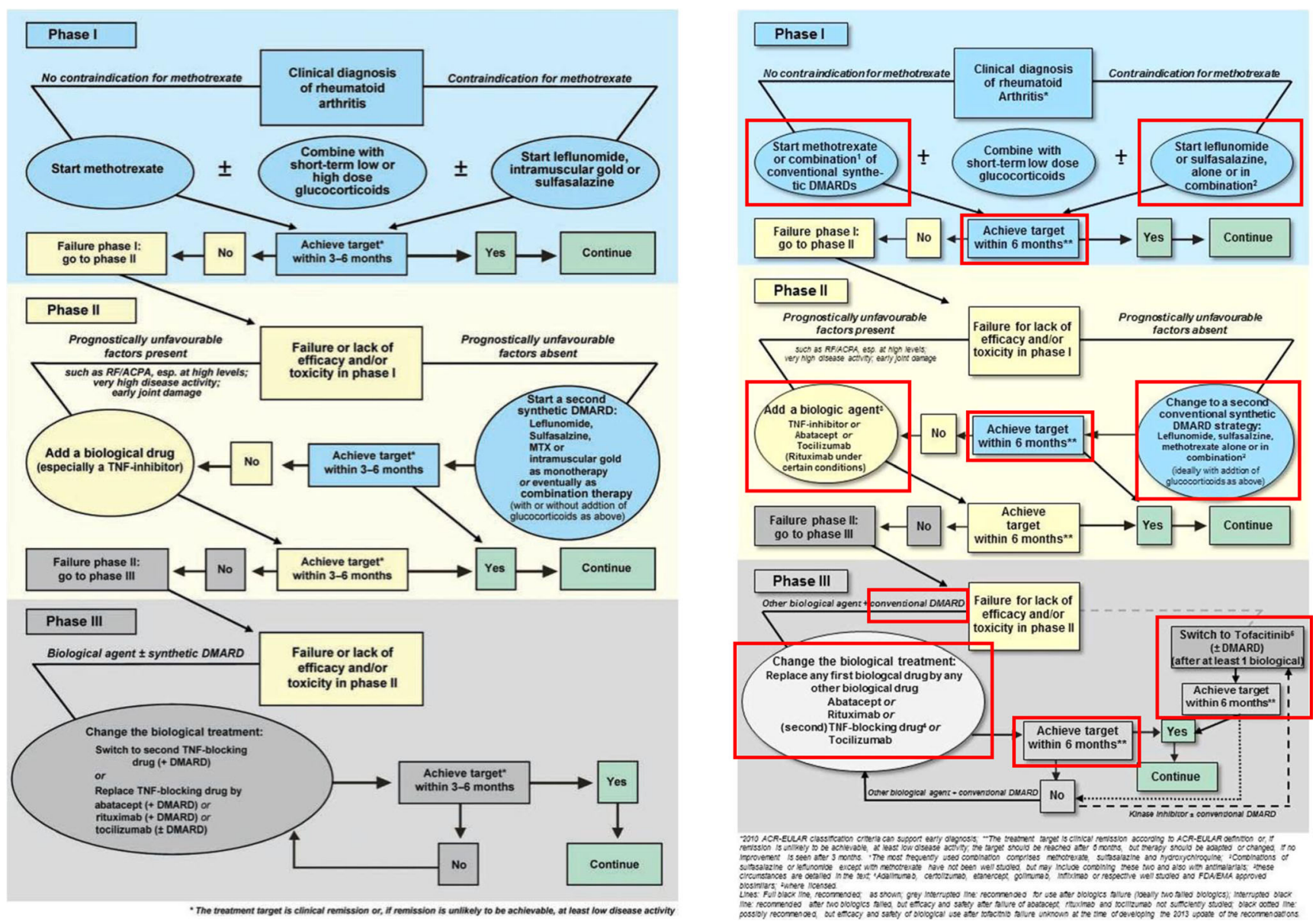

Fig. 1 Comparison of the 2010 and 2013 EULAR treatment algorithms [2, 3]. Red boxes indicate areas of material difference between the guidelines

performed previously to determine whether the applicability and implementation of the EULAR recommendations has changed, both over time and given the recent update. Here we report on the responses from participants in three separate surveys. Group $1(n=84)$ combines the results of surveys conducted with participants at a EULAR recommendations consensus meeting convened in Dubai, United Arab Emirates (UAE), in January 2014 and an expert meeting convened in Marrakech, Morocco, in March 2014. The participants at the UAE meeting, which was chaired by Associate Professor Peter Nash, came from Egypt, Libya, Qatar, Saudi Arabia, Tunisia and the UAE, while the 69 participants at the Morocco meeting came from Algeria, Egypt, Kuwait, Lebanon, Saudi Arabia, Tunisia and the UAE. The participants in group 2 were surveyed during an expert meeting convened in Cape Town, South Africa, during February 2014.

The groups voted on their agreement with and the appropriateness and local applicability of the EULAR recommendations. Group 1 was asked whether they agreed with each recommendation and whether each was applicable to their clinical practice, while group 2 was asked whether they could easily implement each recommendation. Possible answers for each question were strongly disagree, disagree, agree and strongly agree; votes were classified as 'agree' if the response was 'agree' or 'strongly agree' and 'disagree' if the response was 'disagree' or 'strongly disagree'.

\section{Agreement with, applicability of and ease of implementation of the EULAR 2013 recommendations in the Middle East and Africa region}

Figure 2 summarises the responses of group 1 in relation to agreement with and applicability of the EULAR 2013 recommendations and of group 2 in relation to whether these recommendations are easy to implement. Taking the findings of the earlier survey on the EULAR 2010 recommendations into consideration, Fig. 3 shows responses for those recommendations that are unchanged or materially unchanged from 2010 to 2013. It should be noted that the question around implementation differed slightly between the two surveys (2010: "How many rheumatologists implement this recommendation in practice?" 2014: "Is this recommendation easy to implement?"), but the responses are shown to give a broad feel for any potential changes to local practices that may now be more in line with the updated EULAR recommendations. The 


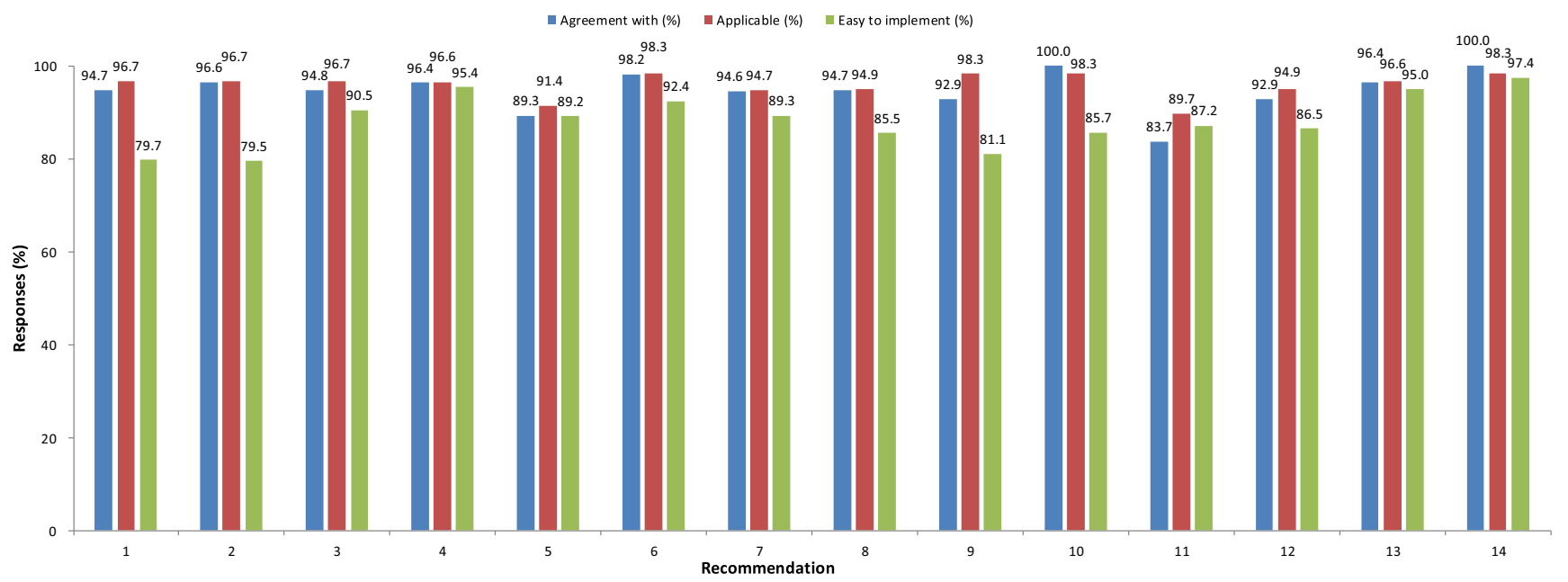

Fig. 2 Responses of new expert groups in relation to agreement with each of the 2013 EULAR recommendations (group 1), acceptability of each recommendation (group 1) and whether each recommendation was easy to implement (\%). Possible answers for each question were strongly

disagree, disagree, agree, strongly agree; votes were classified as 'agree' if the response was 'agree' or 'strongly agree' and 'disagree' if the response was 'disagree' or 'strongly disagree'

(a)

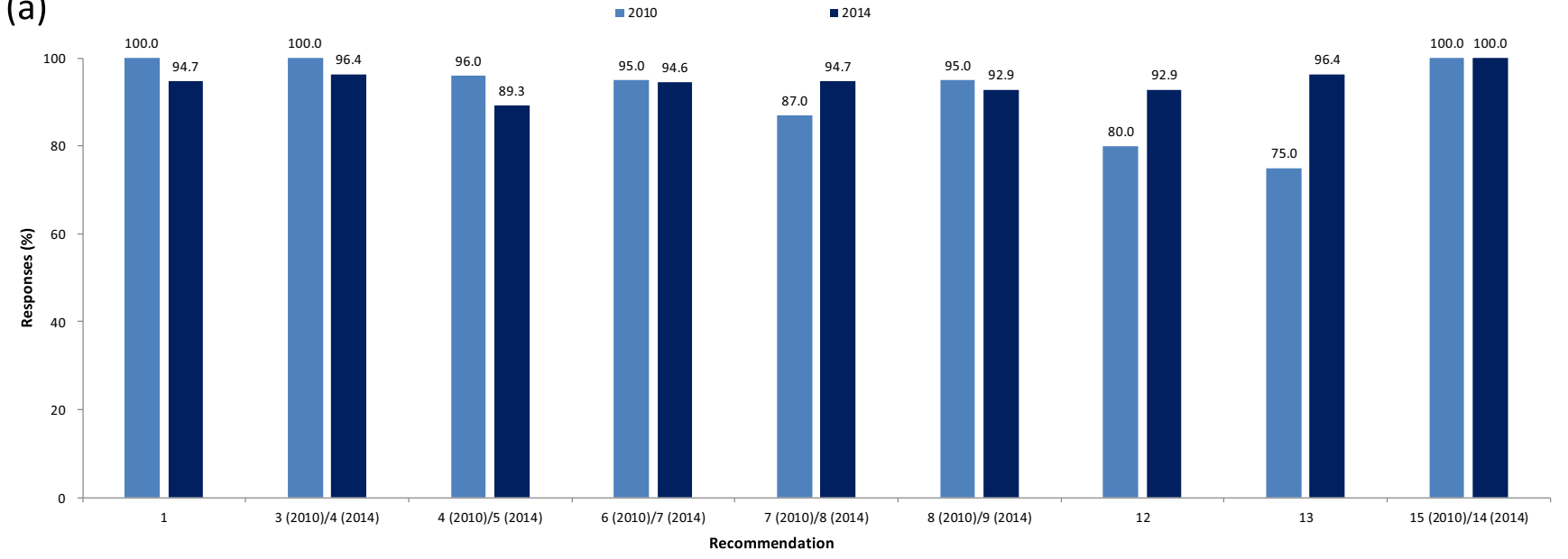

(b)

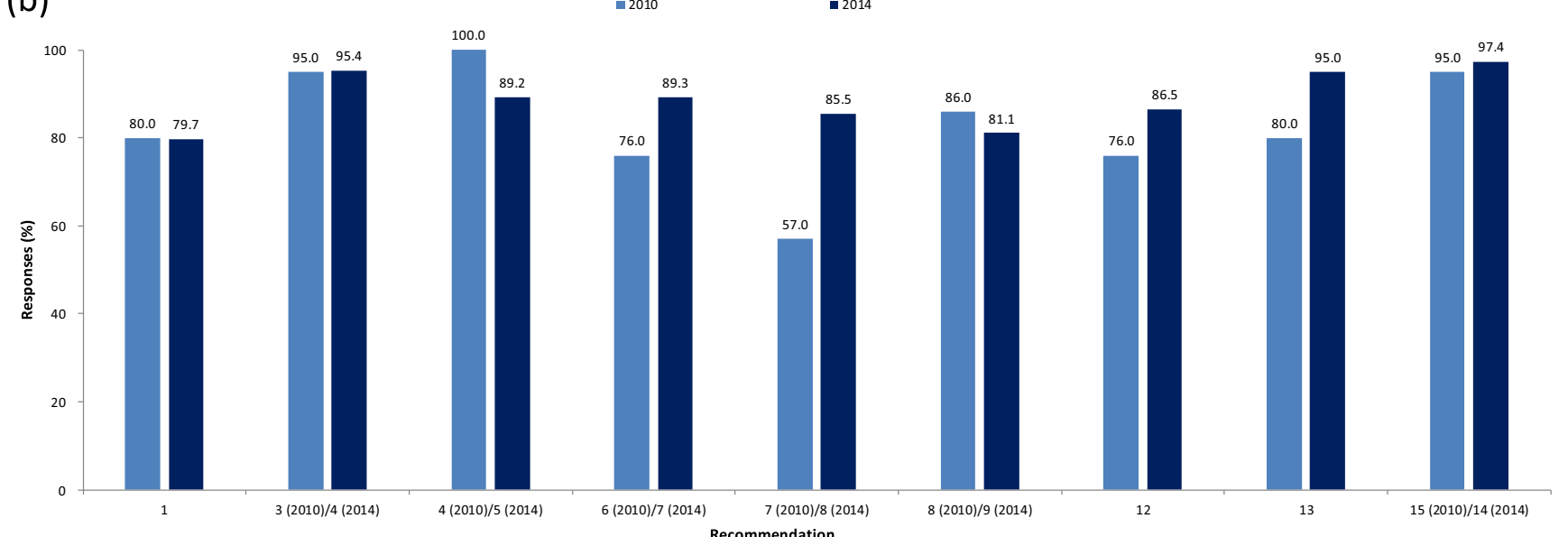

Fig. 3 Comparison of expert groups' responses in relation to agreement with (a) and ease of implementation (b) of EULAR recommendations that were unchanged or broadly unchanged between the 2010 and 2013 guidance. Possible answers for each question were strongly disagree, disagree, agree, strongly agree; votes were classified as 'agree' if the response was 'agree' or 'strongly agree' and 'disagree' if the response was 'disagree' or 'strongly disagree' 
participants were very supportive of the EULAR 2013 recommendations, but these experts had a specific interest in RA and so general awareness among the wider population of other clinicians and stakeholders without a special interest in RA was likely to be lower.

More than $80 \%$ of participants in group 1 agreed or strongly agreed with all of the EULAR recommendations, and more than $90 \%$ of participants in group 2 agreed or strongly agreed that all recommendations (but recommendation 11 - the only entirely new recommendation relating to the janus kinase (JAK) inhibitor tofacitinib) were applicable to their clinical practice. Overall, the proportion of respondents who agreed with the recommendations and who believed that they were applicable to their clinical practice was much higher than the proportion of respondents who agreed or strongly agreed that the recommendation was easy to implement. This reassuringly suggests that rheumatologists in the region still broadly support the EULAR recommendations but highlights that some of the recommendations may remain aspirational in this setting. This may be due to practicalities surrounding implementation in some parts of the region, most often due to issues around access to drugs and monitoring. The perceived ease of implementation was generally higher in our recent survey than in the previous study.

\section{Use of synthetic and biologic DMARDs}

In the previous study, recommendations 7 and 14 were identified as difficult to implement due to problems around access to drugs because of their cost and the associated monitoring [1]. In our recent survey, more respondents agreed with recommendation 7(2010)/8(2013), in which patients who do not achieve treatment target with the first disease-modifying antirheumatic drug (DMARD) are recommended to switch to a second conventional synthetic DMARD or be given an additional biologic DMARD depending on the presence of poor prognostic factors. More respondents also felt that this was easy to implement, which may reflect increased access to these agents. The use of combination therapy with methotrexate (MTX) plus a biologic in patients with poor prognostic factors, the subject of original recommendation 14(2010), is not covered in the same format in the 2013 EULAR recommendations.

Recommendations 1, 6 and 10 were reported as difficult to implement in the previous study because they advocated aggressive management with early use of intensive therapies and tight disease control through frequent monitoring. This may be difficult to achieve due to lack of time to perform measurements of Disease Activity Score (DAS) and Health Assessment Questionnaire (HAQ), lack of awareness, misinformation (for example, about drug toxicity and comorbidities), local practices involving exhausting synthetic DMARDs and glucocorticoids before a biologic is introduced, delayed referral, shortages of specialist and support staff, economic issues (including the need to prioritise funding to diseases with other higher burdens), late access to patients, lack of local data and issues around lack of appropriate insurance in countries with high populations of expats. Agreement with recommendation 1 , which relates to starting DMARDs as soon as the diagnosis of RA is made, was actually lower in our current survey, although the proportions of respondents who felt that this was easy to implement in practice was similarly low in both surveys. Agreement with recommendation 6(2010)/7(2013), which relates to the use of steroids as part of the initial treatment strategy, was almost identical, but participants in our more recent survey felt that it was easier to implement than those in the earlier study, indicating a possible improvement in access to these agents. Recommendation 10(2010) covered the use of synthetic DMARDs such as azathioprine and cyclosporin A in patients with severe refractory RA or contraindications to biologics or other DMARDs, which are no longer recommended by EULAR.

Agreement with recommendation 3(2010)/4(2013), which relates to the use of MTX as the first treatment strategy in patients with active RA, was somewhat lower than in the previous study, but the ease of implementation was broadly similar in the previous study and our recent survey. Agreement with and ease of implementation of recommendation $4(2010) / 5(2013)$, which relates to the use of leflunomide and sulfasalazine (and injectable gold in 2010), were both somewhat lower in the more recent survey. This pattern was also seen for recommendation $8(2010) / 9(2013)$, which relates to the use of biologic DMARDs in patients with insufficient response to MTX and other synthetic DMARDs, as the proportions of participants who agreed with and felt that it was easy to implement were slightly lower in the recent survey. In contrast, more respondents agreed with recommendations 12 and 13, which relate to the use of biologic DMARDs in patients in persistent remission following tapering of glucocorticoids and the cautious titration of synthetic DMARD doses in cases of long-term remission, and felt these recommendations were easy to implement. All participants in both studies agreed with recommendation 9(2010)/10(2013), which relates to strategies following failure of a first antitumour necrosis factor (TNF) inhibitor. The proportion of respondents who felt that this was easy to implement was slightly lower in the more recent survey, which may reflect that the current recommendation specifies more and newer treatment options not yet widely available in this region.

\section{Use of a JAK inhibitor}

Recommendation 11 was the only entirely new recommendation for 2013 and relates to the use of tofacitinib, the biologic 
DMARD most recently added to the rheumatologist's armamentarium, which was not licensed when the previous guidance was published. This was the recommendation with the lowest agreement $(83.7 \%)$, likely due to the fact that this agent is still relatively new to the market. Interestingly, more participants felt that this recommendation was easier to implement than agreed with. The lack of agreement may relate more to the phrasing of this particular recommendation, as the experts believed that waiting for a biologic treatment to fail may not always be applicable and that some doctors may want to go straight to tofacitinib.

\section{Treatment targets and monitoring}

Recommendation 2(2010), which relates to treatment targets and monitoring, has been split into two separate recommendations in 2013: new recommendation 2(2013) covers the treatment target component and new recommendation 3(2013) covers the monitoring aspects. Agreement with the new split recommendations was slightly lower than that for the combined recommendation in the previous study ( 96.6 and $94.8 \%$ for 2(2013) and 3(2013) vs $100 \%$ for recommendation 2(2010)), as was the perceived ease of implementation of new recommendation 3(2013) (90.5 vs $94.0 \%$ ). However, the perceived ease of implementation of new recommendation 2(2013) was dramatically lower than that of original recommendation 2 (79.5 vs $94.0 \%$ ). With the only difference in this revised recommendation being the loss of 'as soon as possible', it is difficult to understand this considerable change, although it may be that experts think it does not go far enough. For example, the Saudi Arabian authors of a recent editorial wanted to treat not to target but to workability to ensure that RA patients remain active working members of Saudi society, which they felt would be a more stringent objective [4]. They noted that this would require the use of a valid patient assessment tool and further research to optimise the implementation of workability measures.

\section{Factors to consider when adjusting treatment}

All participants at both time points agreed with recommendation 15(2010)/14(2013), which relates to the consideration of factors other than disease activity, such as progression of damage, comorbidities and safety concerns, when adjusting treatment. The proportion of participants who felt that this was easy to implement was higher in the more recent survey, which could reflect the more widespread availability of tests required to measure disease progression and increased recognition or reporting of comorbidities.

\section{Discussion}

In general, our results hint at a trend towards less agreement with recommendations around long-term use of conventional DMARDs and steroids and more agreement with those recommending the earlier use of biologic DMARDs. This may reflect a shift in attitudes due to increasing acceptance that more aggressive treatment is required earlier, and agreement that sustained use of conventional DMARDs and steroids is not universally successful in inducing remission, coupled with growing confidence about the efficacy, use and safety of the first-generation biologics in this region. For example, Alawneh et al. reported that RA in Jordan is severe, progressive and associated with comorbidities such as obesity, hypertension and diabetes, noting that remission rates are low despite active treatment with DMARDs, including TNF agents, by rheumatology clinics [5]. Although Slimani et al. found that RA in Algeria may be less aggressive than in Western countries, with more dominant seronegative oligoarthritis forms, remission rates in the two settings were comparable [6]. Lutef et al. found that remission rates in Qatar are better than those reported in other Gulf countries, which may be related to the use of anti-TNFs, but rates are still low when compared to the USA and European countries. Almost one third of their RA patients are not well controlled, and some may have comorbid conditions which limit the use of synthetic and biologic therapies while other patients may have joint damage due to a long-standing disease and if their diseases were acquired in the pre-biologics era [7]. Malemba et al. found continued radiographic progression in Congo despite methotrexate, which was often given in low doses, and need for an additional DMARD; lack of follow-up was also a major issue [8]. Both Hodkinson et al. from South Africa and Ibn Yacoub et al. from Morocco found that RA significantly affects physical and mental aspects of patient's health-related quality of life (HRQoL), with even early RA having a broad impact on HRQoL in indigent South Africans $[9,10]$. Patients in South Africa showed substantial functional disability and suboptimal mental health despite 12 months of DMARD therapy, while in Morocco those taking methotrexate showed improvements in mental health and those taking biologics showed improvements in physical and social domains $[9,10]$. The South African group also found that those who have an inadequate response to traditional DMARDs at 6 months are unlikely to show further improvement on these agents at 12 months, underscoring the need for better disease management through tight control, patient education and biologic therapy [9]. This is reflected in the updated treatment recommendations from South Africa, which advocate early diagnosis, prompt initiation of DMARDs and an intense treatment strategy. This includes assessment of disease activity with composite scores such as the Simplified Disease Activity Index (SDAI), frequent assessments and escalation of therapy 
until low disease activity or remission and the use of synthetic DMARDs alone (in combination with concomitant corticosteroids if necessary) and biologic DMARDs in refractory disease [11].

Mody, reporting from the combined 7th African League of Associations for Rheumatology (AFLAR) and 23rd biennial South African Rheumatism and Arthritis Association (SARA A) congress held in April 2013, noted an encouraging increase in interest in rheumatology, with regular rheumatology congresses and educational meetings held annually or biennially in many of the constituent countries of this region [12]. The increased interest perhaps results from the numerous papers highlighting the suboptimal management of RA in this region and the consequent calls to collect further epidemiological data from across the region to increase understanding of the intra- and intercountry variability of the disease, set up registries to monitor the safety of newer treatments and raise awareness of the impact and burden of this condition among physicians and patients, develop national recommendations for managing RA and monitoring comorbidities, and improve training of HCPs from all relevant disciplines so that they can recognise early inflammatory arthritis and make appropriate referral and fast-track patients to ensure earlier introduction of intensive treatment, thus minimising disease activity as soon as possible after diagnosis [1, 13-17]. Encouragingly, Zafar et al. found that the introduction of support groups, awareness programmes and public campaigns in the UAE in recent years had significantly reduced the time to diagnosis and initiation of DMARD therapy, although they recommended larger studies to substantiate this further and to address whether shorter times to diagnosis and to treatment can positively influence rates of remission and quality of life [18].

Despite promising indications that we are moving in the right direction, challenges remain. The financial barrier to the use of basic tests, imaging, DMARDs (including biologics) and surgery still persists in many resource-poor countries [12, 15, 19]. Alawneh et al. recognised the need to increase documentation of DAS scores when treating patients to allow monitoring of targets to be monitored [5], and Ickinger et al. suggested the use of the rheumatoid arthritis articular damage (RAAD) score in under-resourced settings [20]. Issues around finances are exacerbated by a dearth of rheumatologists to diagnose and manage patients in many countries, with reports that some patients living in the UAE are seeing rheumatologists in the UK to get a diagnosis [12, 15, 19]. Infections were a common theme among the many abstracts at the AFLAR/ SARAA 2013 meeting [12], which ties in with our previous finding that physicians can be wary of using immunosuppressive agents in a population already at risk of endemic infections such as TB [1]. Indeed, Hammoudeh et al. confirmed that the risk for active TB and other infections in patients with RA from Asia, Africa and the Middle East is increased in patients receiving TNF inhibitors and higher in those receiving monoclonal antibodies versus the soluble TNF receptor etanercept [21], which is also highlighted by the South African recommendations [11]. Pettipher et al. reported that infections were a major reason for discontinuation in South Africans and concluded that biologics with alternative modes of action are needed for patients with moderate to severe RA in South Africa [22]. From a more positive viewpoint, Tikly et al. noted that mandatory screening for latent TB infection prior to initiation of TNF inhibitors has markedly reduced the incidence of TB in treated patients, but with reactivation of TB still occurring in some patients, they suggest considering nonTNF inhibitor biologics ahead of TNF inhibitors in those with DMARD-resistant disease [17].

\section{Conclusion}

In conclusion, comparison of these two reports on the applicability and implementation of the EULAR recommendations and recent papers in the literature suggest that improvements in the management of RA in the Middle East and Africa have been made, but challenges still remain. It therefore is important to maintain the current momentum, as further work raising awareness, increasing education, ensuring early diagnosis and more aggressive management and increasing the number of available specialists is needed before the management of RA throughout this region can be considered optimal.

Acknowledgments This paper was based on the proceedings of three advisory boards, which were funded by Pfizer.

Disclosures None.

\section{References}

1. El Zorkany B, Alwahshi HA, Hammoudeh M, Al Emadi S, Benitha R, Al Awadhi A, Bouajina E, Laatar A, El Badawy S, Al Badi M, AlMaini M, Al Saleh J, Alswailem R, Ally MM, Batha W, Djoudi H, El Garf A, El Hadidi K, El Marzouqi M, Hadidi M, Maharaj AB, Masri AF, Mofti A, Nahar I, Pettipher CA, Spargo CE, Emery P (2013) Suboptimal management of rheumatoid arthritis in the Middle East and Africa: could the EULAR recommendations be the start of a solution? Clin Rheumatol 32:151-159

2. Smolen JS, Landewe R, Breedveld FC, Dougados M, Emery P, Gaujoux-Viala C, Gorter S, Knevel R, Nam J, Schoels M, Aletaha D, Buch M, Gossec L, Huizinga T, Bijlsma JW, Burmester G, Combe B, Cutolo M, Gabay C, Gomez-Reino J, Kouloumas M, Kvien TK, Martin-Mola E, McInnes I, Pavelka K, van Riel P, Scholte M, Scott DL, Sokka T, Valesini G, van Vollenhoven R, Winthrop KL, Wong J, Zink A, van der Heijde D (2010) EULAR recommendations for the management of rheumatoid arthritis with synthetic and biological disease-modifying antirheumatic drugs. Ann Rheum Dis 69:964-975

3. Smolen JS, Landewe R, Breedveld FC, Buch M, Burmester G, Dougados M, Emery P, Gaujoux-Viala C, Gossec L, Nam J, Ramiro S, Winthrop K, de Wit M, Aletaha D, Betteridge N, Bijlsma JW, Boers M, Buttgereit F, Combe B, Cutolo M, 
Damjanov N, Hazes JM, Kouloumas M, Kvien TK, Mariette X, Pavelka K, van Riel PL, Rubbert-Roth A, Scholte-Voshaar M, Scott DL, Sokka-Isler T, Wong JB, van der Heijde D (2014) EULAR recommendations for the management of rheumatoid arthritis with synthetic and biological disease-modifying antirheumatic drugs: 2013 update. Ann Rheum Dis 73:492-509

4. Almoallim H, Kamil A (2013) Rheumatoid arthritis: should we shift the focus from "Treat to Target" to "Treat to Work?". Clin Rheumatol 32:285-287

5. Alawneh KM, Khassawneh BY, Ayesh MH, Smadi M (2014) Rheumatoid arthritis in Jordan: a cross sectional study of disease severity and associated comorbidities. Ther Clin Risk Manag 10: 363-366

6. Slimani S, Abbas A, Ben Ammar A, Kebaili D, Ali EH, Rahal F, Khamari MC, Baltache A, Khider I, Chiheub R, Khelif K, Akbi S, Rahmani S, Dahou-Makhloufi C, Brahimi-Mazouni N, AbtrounBenmadi S, Ladjouze-Rezig A (2014) Characteristics of rheumatoid arthritis in Algeria: a multicenter study. Rheumatol Int

7. Lutf A, Poil AR, Hammoudeh M (2014) Characteristics of patients with rheumatoid arthritis in Qatar: a cross-sectional study. Int J Rheum Dis 17:63-65

8. Malemba JJ, Mbuyi Muamba JM, Mukaya J, Bossuyt X, Verschueren P, Westhovens R (2013) Treatment of rheumatoid arthritis with methotrexate in Congolese patients. Clin Rheumatol 32: 1323-1327

9. Hodkinson B, Musenge E, Ally M, Meyer PW, Anderson R, Tikly M (2012) Functional disability and health-related quality of life in South Africans with early rheumatoid arthritis. Scand J Rheumatol 41:366374

10. Ibn Yacoub Y, Amine B, Laatiris A, Hajjaj-Hassouni N (2012) Health-related quality of life in Moroccan patients with rheumatoid arthritis. Clin Rheumatol 31:1471-1477

11. Hodkinson B, Van Duuren E, Pettipher C, Kalla A, South African R, Arthritis A (2013) South African recommendations for the management of rheumatoid arthritis: an algorithm for the standard of care in 2013. S Afr Med J 103:576-585

12. Mody GM (2013) AFLAR and SARAA 2013 Congress-a watershed event for rheumatology in Africa. Clin Rheumatol 32:925-928

13. Dowman B, Campbell RM, Zgaga L, Adeloye D, Chan KY (2012) Estimating the burden of rheumatoid arthritis in Africa: a systematic analysis. J Glob Health 2:020406
14. Halabi H, Alarfaj A, Alawneh K, Alballa S, Alsaeid K, Badsha H, Benitha R, Bouajina E, Al Emadi S, El Garf A, El Hadidi K, Laatar A, Makhloufi CD, Masri AF, Menassa J, Al Shaikh A, Swailem RA, Dougados M (2014) Challenges and opportunities in the early diagnosis and optimal management of rheumatoid arthritis in Africa and the Middle East. Int J Rheum Dis. doi:10.1111/1756-1185X.12320

15. Hodkinson B, Tikly M, Adebajo A (2014) Rheumatoid arthritis in the developing world: stepping up to the challenge. Clin Rheumatol. doi: 10.1007/s10067-10014-12690-10063

16. Hajjaj-Hassouni N, Al-Badi M, Al-Heresh A, Al-Emadi S, El Bawendi A, El Garf A, El Hadidi K, Halabi H, Hammoudeh M, El Hassani S, Al Maaini M, Nahar I, Ladjouze Rezig A, Sellami S, Sweiri W, Alswailem R, Traub B, Uthman I, van Duuren E, Zakraoui L, El Zorkany B, Carmona L, Dougados M (2012) The practical value of biologics registries in Africa and Middle East: challenges and opportunities. Clin Rheumatol 31:407-416

17. Tikly M, Hodkinson B, Dheda K (2014) Biologic therapy for rheumatoid arthritis in developing countries - a place for non-TNF inhibitors as first-line treatment? Rheumatology (Oxford)

18. Zafar S, Badsha H, Mofti A, Delosantos A, Altares J, Matudio G, Kong KO (2012) Efforts to increase public awareness may result in more timely diagnosis of rheumatoid arthritis. J Clin Rheumatol 18: 279-282

19. Saberi M (2013) Arthritis in UAE: diagnose disease early. In. Gulfnews.com. Dubai, UAE: Al Nisr Publishing LLC. Available from: http://gulfnews.com/life-style/health/arthritis-in-uae-diagnosedisease-early-1.1207430

20. Ickinger C, Musenge E, Tikly M (2013) Patterns and predictors of joint damage as assessed by the rheumatoid arthritis articular damage (RAAD) score in South Africans with established rheumatoid arthritis. Clin Rheumatol 32:1711-1717

21. Hammoudeh M, Alarfaj A, Chen DY, Djoudi H, Youseif E, Zhu J (2013) Safety of tumor necrosis factor inhibitors use for rheumatoid arthritis and ankylosing spondylitis in Africa, the Middle East, and Asia: focus on severe infections and tuberculosis. Clin Rheumatol 32: 293-300

22. Pettipher C, Rudolph R, Musenge E, Tikly M (2014) A prospective study of anti-tumor necrosis factor therapy in South African rheumatoid arthritis patients. Int J Rheum Dis. doi:10.1111/1756-1185X. 12299 